\title{
Magnetic X-ray Measurements Using the Elliptical Multipole Wiggler
}

\author{
P. A. Montano ${ }^{1,2}$, Yinwan $\mathrm{Li}^{1,2}$, M. A. Beno ${ }^{1}$, G. Jennings ${ }^{1}$, and C. W. Kimball ${ }^{1.3}$
}

1. Materials Science Division, Argonne National Laboratory, Argonne, IL 60439

2. Department of Physics, Uinversity of llinois at Chicago, Chicago, IL 60680

3. Department of Physics, Northern Illinois University, DeKalb, IL 60115

\section{Materials Science Division \\ Argonne National Laboratory \\ Argonne, IL 60439}

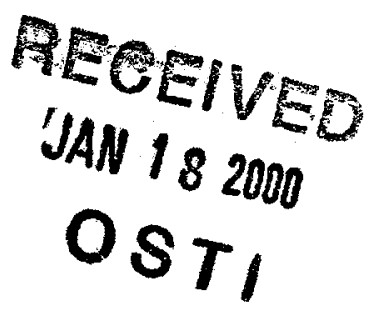

October 1999

jjc

Distribution:

1-2. PRS

3. J. M. Gibson

4. P. A. Montano

5. F. Y. Fradin

6. Office of Science

7. Editorial Office

8. Authors

Submitted for publication in the Proceedings of SPIE $44^{\text {th }}$ Annual Meeting, Denver, CO, July 18-23, 1999.

This work is supported by the Division of Materials Sciences, Office of Basic Energy Sciences of DOE, under contract No. W-31-109-ENG-38. 


\section{DISCLAIMER}

This report was prepared as an account of work sponsored by an agency of the United States Government. Neither the United States Government nor any agency thereof, nor any of their employees, make any warranty, express or implied, or assumes any legal liability or responsibility for the accuracy, completeness, or usefuiness of any information, apparatus, product, or process disclosed, or represents that its use would not infringe privately owned rights. Reference herein to any specific commercial product, process, or service by trade name, trademark, manufacturer, or otherwise does not necessarily constitute or imply its endorsement, recommendation, or favoring by the United States Government or any agency thereof. The views and opinions of authors expressed herein do not necessarily state or reflect those of the United States Government or any agency thereof. 


\section{DISCLAIMER}

Portions of this document may be illegible in electronic image products. Images are produced from the best available original document. 


\title{
Magnetic X-Ray Measurements Using the Elliptical Multipole
}

\section{Wiggler}

\author{
P.A. Montano ${ }^{1,2}$,Yinwan Li ${ }^{1,2}$, Uta Ruett $^{1,3}$, M.A. Beno ${ }^{1}$, G. Jennings ${ }^{1}$, and C.W. Kimball ${ }^{1,3}$
}

1. Materials Science Division, Argonne National Laboratory, Argonne IL 60439.

2. Department of Physics, University of Illinois-Chicago, Chicago IL 60680.

3. Department of Physics, Northern Illinois University, DeKalb IL 60115

\section{$\underline{\text { Abstract }}$}

The EMW at the BESSRC beam lines at the APS provides high photon flux at high energies with the capability of producing circular polarization on axis. We observe a high degree of circularly polarized x-rays at such energies. The polarization and frequency tunability of the elliptical multipole wiggler (EMW) is an ideal source for many magnetic measurements from X-ray Magnetic Circular Dichroism (XMCD) to Compton scattering experiments. We performed Compton scattering measurements to determine the polarization and photon flux at the sample as a function of the deflection parameters $K_{y}$ and $K_{x}$. We used for our measurements a Si (220) Laue monochromator providing simultaneous photon energies at $50 \mathrm{keV}, 100 \mathrm{keV}$ and $150 \mathrm{keV}$. Magnetic Compton Profiles were determined by either switching the magnet polarity or the photon helicity. The results obtained using $\mathrm{Fe}(110)$ single crystals were very similar.

Keywords: Compton scattering, polarization, insertion device, elliptical multipole wiggler, $\mathrm{x}$ rays, synchrotron, flux, magnetic, helicity.. 


\section{Introduction}

Magnetic Compton scattering (MCS) experiments ${ }^{1,2}$ require high photon flux at high $x$-ray energies and a high degree of circular polarization. The Advanced Photon Source (APS) is an excellent source of high energy x-rays providing an ideal environment for MCS experiments. Quarter-wave plates and bending magnetic radiation observed off the bending plane were the most frequently used sources of circularly polarized $x$-rays ${ }^{3,4,5}$. In recent years, insertion devices like the asymmetric wiggler $(\mathrm{AW})^{6}$ and the elliptical multipole wiggler (EMW) ${ }^{\mathbf{7 , 8 , 9}}$ capable of producing circularly polarized high energy x-rays are frequently utilized for MCS experiments. In addition to the need for high photon intensities at high energies, as well as a high degree of circular polarization (switching polarization is preferable to switching the magnetic field), MCS experiments require high momentum resolution and efficient detection.

In this paper we describe the EMW insertion device ${ }^{10}$ installed in sector 11 at the APS (Basic Energy Sciences Synchrotron Radiation Center (BESSRC) CAT) which is ideally suited for high-energy scattering, and show some of the measurements performed with this device. This device is capable of delivering circularly polarized $x$-rays on axis. The polarization and frequency tunability of the elliptical multipole wiggler (EMW) is very suitable for Compton scattering experiments. It facilitates performance of experiments with high resolution and good signal to noise. We have characterized the performance of the insertion device by measuring the polarization and photon flux at the Compton Scattering experimental station.

\section{Experimental}

\subsection{Insertion Device}

The circularly polarized high energy $\mathrm{x}$-rays are produced by the elliptical multipole wiggler. The EMW vertical magnetic field is produced by 36 poles of which 32 are full field, the magnetic structure has a period length of $16 \mathrm{~cm}$. We can achieve a $\mathrm{K}$ y of14.4 at the lowest gap $(24 \mathrm{~mm})$. The horizontal magnetic field is achieved by using electromagnets, 36 poles of which 32 are full field. The EMW electromagnet poles are fabricated from laminated iron in order to operate with a switching frequency up to $10 \mathrm{~Hz}$. One can change the horizontal field from 0 up to close to $1 \mathrm{~T}$. We 
have measured up to a $\mathrm{K}_{\mathrm{x}}$ of about 1.15 that corresponds to a current of $900 \mathrm{~A}$ in the electromagnets. The device total length is $2.8 \mathrm{~m}$. Figure 1 shows the calculated pattern at $32 \mathrm{keV}$ and $30 \mathrm{~m}$ from the source with $\mathrm{K}_{\mathrm{y}}=14.4$ and $\mathrm{K}_{\mathrm{x}}=1$. This calculation was performed using the characteristic parameters of the synchrotron source in the straight section. The superimposed

numbers are photons/(sec- $\left.\mathrm{mm}^{2}\right)$. One can easily see the lower photon intensity in the center, where the circularly polarized $\mathrm{x}$-rays are found. The fact that the beam splits vertically is utilized to divide the beam into three stations with the center portion going into the MCS experimental station. The MCS station is located at $50 \mathrm{~m}$ from the source.

\subsection{Monochromator}

The monochromator used for the Compton scattering experiments is of the Laue type. A Si (220) annealed crystal $10 \mathrm{~mm}$ wide and $10 \mathrm{~mm}$ thick is mounted on a $\mathrm{Ni}$ plated $\mathrm{Cu}$ support on $\mathrm{Ga}$. The crystal is water-cooled. In addition a water-cooled $\mathrm{Cu}$ filter is mounted in front of the crystal to reduce the heat load. The horizontal acceptance angle is about $1 / 3$ of a mrad. Figure 2 shows a schematic diagram of the monochromator chamber, it can be evacuated or kept in a He atmosphere. The second beam shown in the figure (second monochromator) goes to the highenergy scattering station. The monochromator is mounted in a goniometer with _ and _ motions; in addition it has horizontal and vertical translational degrees of freedom. The MCS monochromator accepts the central part of the beam and part of bottom lobe, consequently slits in front of the MCS station has to be used to reduce or eliminate the bottom lobe of the beam (linearly polarized $\mathrm{x}$-rays).

\subsection{Experimental Measurements}

Beam Profile: We used a half a millimeter vertical slit to profile the beam in the MCS experimental station. A perfect $\mathrm{Si}(220)$ Laue monochromator was used for these measurements with a $2 \mathrm{~mm}$ wide beam impinging on the crystal, the full vertical beam was taken by the crystal. 
We studied the beam profile as a function of $K_{x}$ for a $K_{y}=14.4$. Figure 3 is a representative measurement at $\mathrm{K}_{\mathrm{x}}=1.03$. The smaller intensity at $50 \mathrm{keV}$ is due to the absorption of the filters and the monochromator crystal, the small intensity at $150 \mathrm{keV}$ is to a great extend due to the limited detector efficiency $(\mathrm{Ge})$. One can easily observe that the intensity is reduced at the center, where the circular polarization is dominant. We found excellent agreement between the beam splitting predicted by the EMW theoretical calculations and our measurements.

Compton Scattering: We used Compton Scattering and Magnetic Compton Scattering to determine the photon flux impinging on our sample and the degree of circular polarization. The partial Compton scattering cross section at energies significantly lower than $\mathrm{mc}^{2}$ is given by ${ }^{11,12}$ :

$$
\frac{\mathbf{d} \sigma}{\mathbf{d} \Omega \mathbf{d E} E^{\prime}}=\frac{\mathbf{N m}}{\mathbf{k} \hbar^{2}}\left[\left(\frac{\mathrm{d} \sigma}{\mathrm{d} \Omega}\right)_{\text {charge }} \mathrm{J}\left(\mathbf{p}_{\mathbf{z}}\right)+\left(\frac{\mathrm{d} \sigma}{\mathrm{d} \Omega}\right)_{\operatorname{mag}} \mathrm{J}_{\operatorname{spin}}\left(\mathbf{p}_{\mathbf{z}}\right)\right]
$$

Where,

$$
\begin{aligned}
& \left(\frac{d \sigma}{d \Omega}\right) \text { charge }=\frac{1}{2} \mathbf{r}_{0}^{2}\left(1+\cos ^{2} \Theta\right) \\
& \left.\frac{d \sigma}{d \Omega}\right) m a g=-r_{0}^{2} \frac{E}{m^{2}} P_{c}(1-\cos \Theta)\left\{\left(\hat{q} \cos \Theta+\hat{q}^{\prime}\right) \cdot \hat{m}\right\}
\end{aligned}
$$

$\mathbf{m}$ is a unit vector in the direction of the magnetic axis, _ is the scattering angle, $\mathbf{r}_{\mathbf{0}}$ is the Thompson radius and $\mathrm{P}_{\mathrm{c}}$ is the degree of circular polarization.

$$
N J_{\operatorname{spin}}\left(p_{z}\right)=\frac{1}{2} \iint\left[\rho_{\uparrow}(p)-\rho_{\downarrow}(p)\right] d p_{x} d p_{y}
$$

The Magnetic Compton profile is given by the difference between spins parallel and anti parallel to m. 
We used for the MCS measurements an electromagnet with a maximum field of one Tesla. The photon flux on the sample was measured using the total Compton scattering. The flux measurements were performed using either a perfect $\mathrm{Si}(220)$ Laue crystal or an annealed crystal. The latter gives a significantly higher flux at the sample due to the mosaicity, a factor of 20 to 25 more than the perfect crystal. Figure 4 shows the flux at the sample per mrad measured using a perfect Laue crystal and $\mathrm{K}_{\mathrm{x}}=0, \mathrm{~K}_{\mathrm{y}}=14.4$, (annealed crystal provides a factor of 20/25 higher flux) the vertical slit was $1 \mathrm{~mm}$.

The circular polarization was measured using an $\mathrm{Fe}(110)$ sample at room temperature. The magnetic properties of Fe are well known and MCS has been performed extensively on this material ${ }^{13,14,15}$. Consequently, we selected iron as our standard to calculate $P_{c}$. Figure 5 shows the variation in polarization as a function of $\mathrm{K}_{\mathrm{x}}$ for $\mathrm{K}_{\mathrm{y}}=14.4$ at an energy of $100 \mathrm{keV}$. We used a nine element Ge solid state detector for these measurements, the magnetic field was flipped during this experiment and the scattering angle was $170^{\circ}$. We have to open the slits slightly (optimization of $\mathrm{P}_{\mathrm{c}}{ }^{1 / 2}$ ) in order to obtain good signal to noise. The measurements were performed with an annealed crystal monochromator. The momentum resolution achieved with this detector and geometry was around 0.65 a.u. at $100 \mathrm{keV}$. The photon flux impinging on the sample at $K_{x}=1$ was around $10^{11}$.

The intensity of the impinging photon beam is significantly reduced as a function of $\mathrm{K}_{\mathrm{y}}$, this effect is of course more significant at energies above $50 \mathrm{keV}$. Figure 5 shows the measured flux on the sample for $K_{y}=9$ as a function of $K_{x}$, for $100 \mathrm{keV}$ photons. At $K_{x}=1$ we have two orders of magnitude less photons than fior $\mathrm{K}_{\mathrm{y}}=14.4$, in addition the degree of circular polarization is reduced to about 0.35 .

One of the great advantages of the EMW at the BESSRC beam lines is the possibility of switching the device polarization as a function of time. This is a very important characteristic that can be extremely valuable when working at high magnetic fields, where fast switching of the field is very difficult (for example when using superconducting magnets). We performed a series of measurements using either the flipping magnetic field mode or the flipping helicity mode. The sequence in our measurements is always ABBA, either for the magnet or the EMW polarization. Figure 6 shows the MCP of Fe1 10 for $K_{y}=14.4$ and flipping the photon polarization $\left(K_{x}=1\right.$ to $K_{x}=$ 1), and Figure 7 shows the profile for the case where the magnet field polarity was flipping $\left(\mathrm{K}_{\mathrm{x}}=1\right)$. 
The detector amplifier gains setting were different for the two measurements; consequently the number of points is different. One can clearly observe that both modes of operation give similar results.

We have also used this beam line to perform measurements in more challenging samples like $\mathrm{NiCu}$ alloys, $\mathrm{La}_{0.7} \mathrm{Sr}_{0.3} \mathrm{MnO}_{3}$ single crystals and $\mathrm{Fe}_{7} \mathrm{~S}_{8}$. The measurements are presently being analyzed. As an example, we show in figure 9 the $\mathrm{MCP}$ of $\mathrm{Fe}_{7} \mathrm{~S}_{8}$, the measurements were performed using a $\mathrm{K}_{\mathrm{y}}=9$, (due to heat loading problems in other components of sector 11). $\mathrm{Fe}_{7} \mathrm{~S}_{8}$ is a monoclinic (pseudo-hexagonal) crystal with ferrimagnetic properties $\left(\mathrm{T}_{\mathrm{c}}=578 \mathrm{~K}\right)$ and metallic conduction. The spin direction at room temperature is perpendicular to the c-axis, the spin direction changes as the temperature is lowered. It is known that the magnetic properties in this crystal have significant contributions from the orbitals. Our measurements were performed at room temperature and at an angle of $45^{\circ}$ with the c-axis, we did not achieve full saturation, however a $\mathrm{MCP}$ was observed. We are presently continuing with the study of $\mathrm{Fe}_{7} \mathrm{~S}_{8}$ by measuring the $\mathrm{MCP}$ as a function of temperature.

We find no need to increase the photon flux at the present time since the detectors can not handle higher counting rates, so the Laue $\mathrm{Si}(220)$ crystal satisfies our present needs. We plan to introduce a Bragg focusing optics capable of accepting one milli-radian of beam in the near future, this will require the use of a crystal analyzer instead of solid state detectors. This type of experimental set up will be capable of providing higher momentum resolution, at least better than 0.1 a.u.

\section{Conclusions}

We have shown that EMW at the BESSRC beam lines at the APS provides high photon flux at high energies with the capability of producing circular polarization on axis. Polarization and photon flux on the sample were characterized as a function of the EMW deflection parameters.

We performed MCP measurements by flipping either the EMW polarization or the magnet field direction on the sample. We performed MCP measurements in various magnetic crystals and were able to observe the profiles even for samples with small fraction of magnetic electrons. The second phase of this beam line will incorporate a Bragg focusing monochromator. This will facilitate high momentum resolution MCP measurements. 
Acknowledgments. We want to acknowledge the financial support from the US DOE (BES W-31109-ENG-38) and the State of Illinois under HECA.

\section{References}

1. P.M. Platzman and N. Tzoar, Phys. Rev. B2, 3556 (1970).

2. M. J. Cooper, Rept. Prog. Phys. 48,415 (1985).

3 N. Sakai and K. Ono, Phys. Rev. Lett. 37, 351 (1976).

4 M. J. Cooper et al, Nature 333, 151 (1988).

5.D. M. Mills, Rapid Comm. Phys. Rev. B Vol 36,No11, 6178 (1987).

6.J. Goulon, P. Elleaume and D. Raoux, Nucl. Instr. Methods A 254, 192 (1987); J. Pflueger and G. Heintze, Nucl. Instr. Methods A289, 300(1990).

7.S. Yamamoto, H. Kawata, H. Kitamura, M. Ando, PRL. 62 (1989) 2672.

8.H. Kitamura and S. Yamamoto, Rev. Sci. Instr. 63, 1104, (1991).

9. H. Kawata, T. Miyahara, S. Yamomoto, T. Shioya, H. Kitamura, S. Sato, S. Asaoka, N. Kanaya, A. Lida, A. Mikuni, M. Sato, T. Iwazumi, Y. Katijima, M. Ando, Rev. Sci. Instr. 60 (1989) 1885.

10. P. A. Montano, G. S. Knapp, G. Jennings, E. Gluskin, E. Trakhtenberg, I.B. Vasserman, P. M. Ivanov, D. Frachon, E. R. Moog, L. R. Turner, G. K. Shenoy, M. J. Bedzyk, M. Ramanathan, M. A. Beno, P.L. Cowan, Review of Scientific Instruments, 66,1839(1995).

11. W . Schulke, Chapter 15, 565, Handbook on Synchrotron Radiation, Vol.3 edited by G. Brown and D.E. Moncton, Elsevier Science Publishers B.V. (1991) and references there in.

12. J. E. McCarthy, M. J. Cooper, P. K. Lawson, D. N. Timms, S. O. Manninen, K. Hamalainen and P. Suortti, J. Synchrotron Radiation 4, 102 (1997).

13. S. Yamamoto, H. Kawata, H. Kitamura, M. Ando, N. Sakai and N. Shiotani. Phys. Rev. Lett. 62, 2672 (1889).

14. N. Sakai J. Appl. Cryst. 29, 81 (1996).

15. Y. Kubo and S. Asano, Phys. Rev. B 42, 4431 (1990). 


\section{Figure Captions.}

Figure 1. Intensity distribution as a function of vertical and horizontal positions for $K_{y}=14.4$, $\mathrm{K}_{\mathrm{x}}=1(32 \mathrm{keV})$ at a distance of $30 \mathrm{~m}$ from the source. The numbers are Photons $/\left(\mathrm{sec}-\mathrm{mm}^{2}\right.$ $0.1 \mathrm{BW}$ ).

Figure 2. Schematic diagram of the Laue monochromators.

Figure 3. Beam profile at the MCS experimental station using $0.5 \mathrm{~mm}$ vertical slits.

Figure 4. Photons/(sec-mrad) vs energy for a perfect $\mathrm{Si}(220)$ crystal monochromator at $\mathrm{K}_{\mathrm{y}}=14.4$ and $\mathrm{K}_{\mathrm{x}}=0$.

Figure 5. Degree of circular polarization as a function of $K_{x}$ for $100 \mathrm{keV}$ photons, $K_{y}=14.4$.

Figure 6. Photons/(sec-mrad) vs $K_{x}$ at $100 \mathrm{keV}, K_{y}=9$.

Figure 7. MCP of $\mathrm{Fe}(110)$ at $\mathrm{K}_{\mathrm{y}}=14.4$ obtained by flipping the emw helicity $\left(\mathrm{K}_{\mathrm{x}}=+1\right.$ to $\left.\mathrm{K}_{\mathrm{x}}=-1\right)$.

Figure 8. $\mathrm{MCP}$ of $\mathrm{Fe}(110)$ at $\mathrm{Ky}=14.4$ and $\mathrm{Kx}=1$ obtained by flipping the magnetic field polarity $(+0.8$ to $-0.8 \mathrm{~T})$.

Figure 9. $\mathrm{MCP}$ of $\mathrm{Fe}_{7} \mathrm{~S}_{8}$ obtained at room temperature with $\mathrm{K}_{\mathrm{y}}=9$ and $\mathrm{K}_{\mathrm{x}}=1.15$, the incident energy was $100 \mathrm{keV}$. 

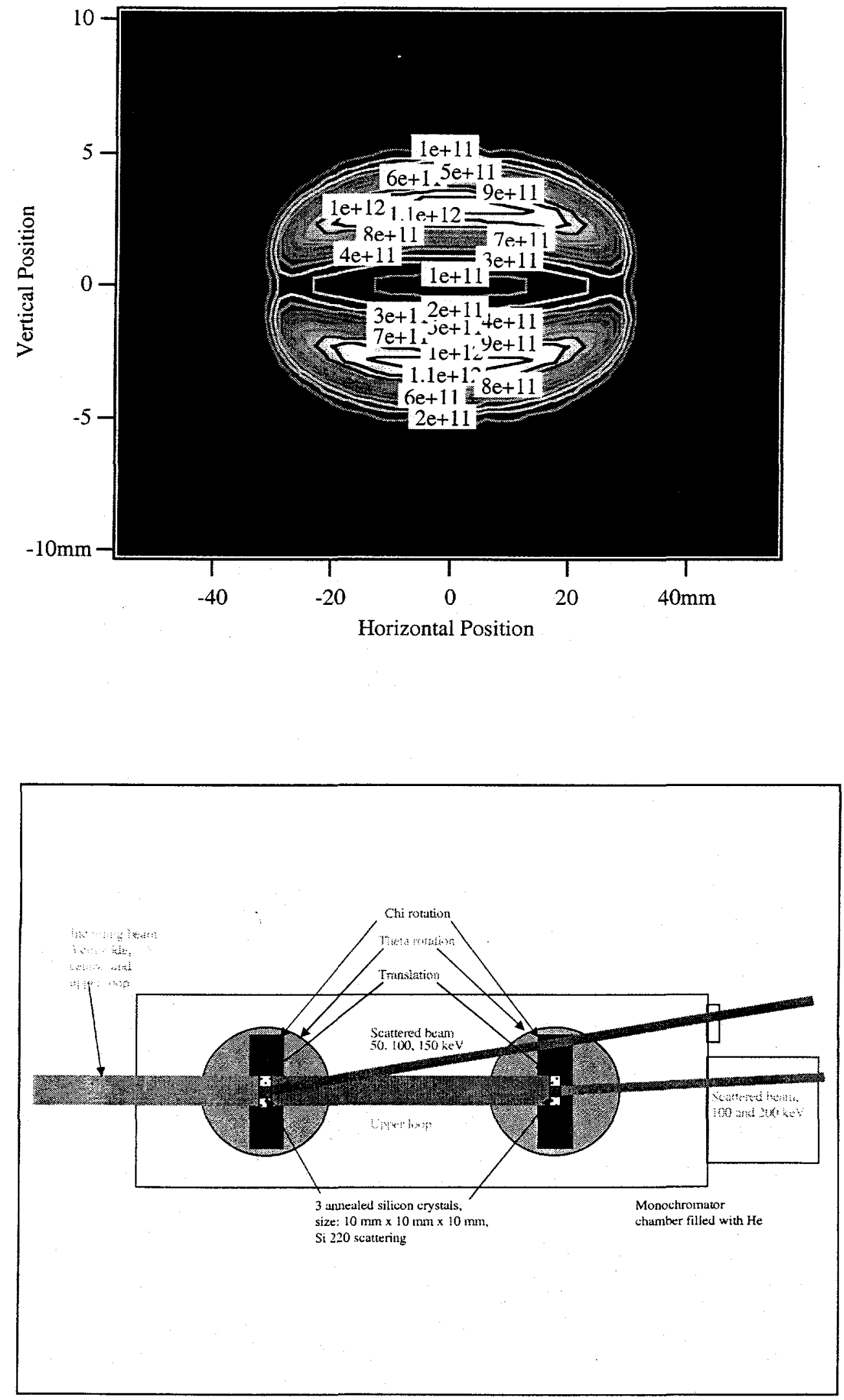

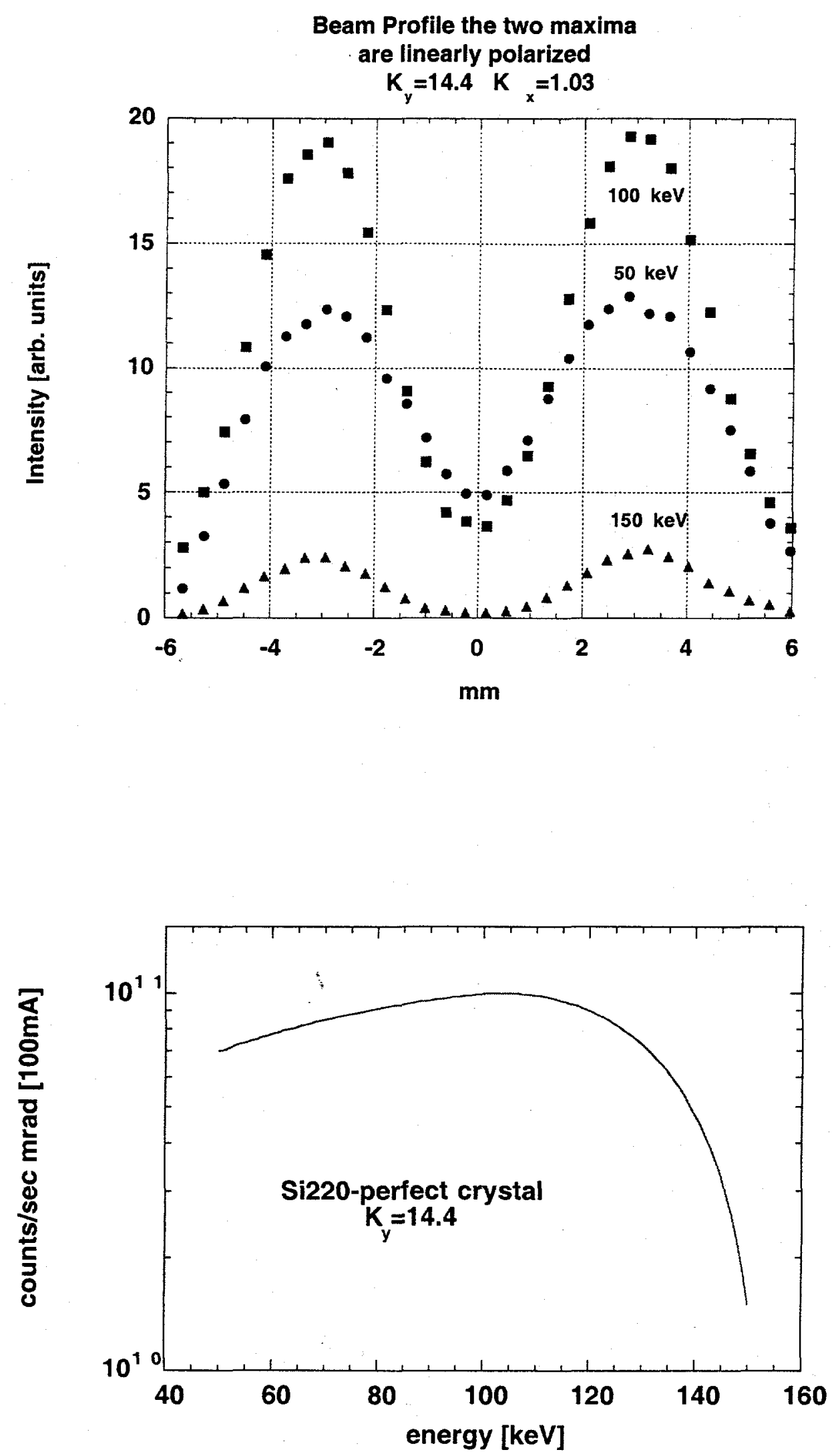

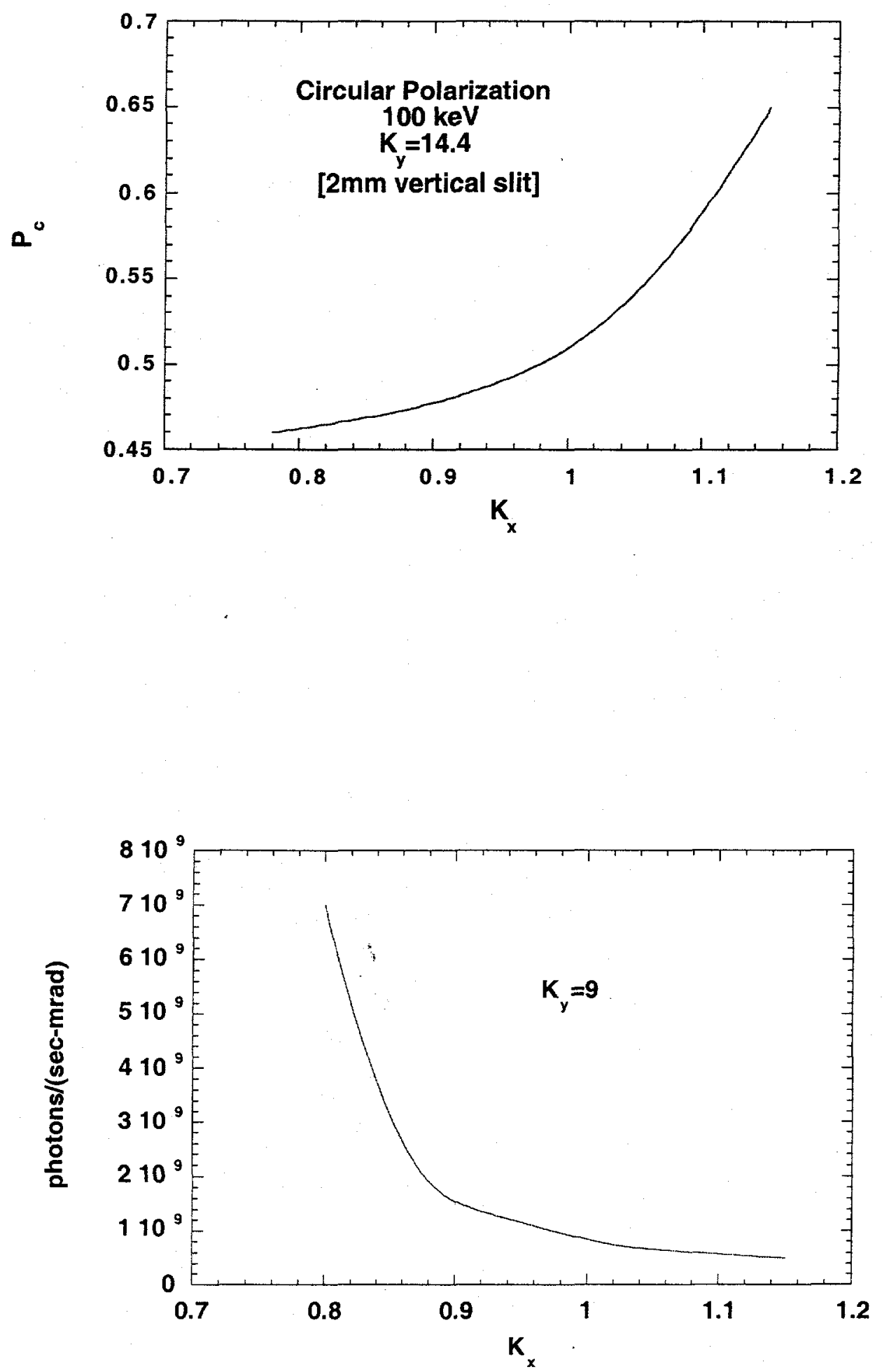

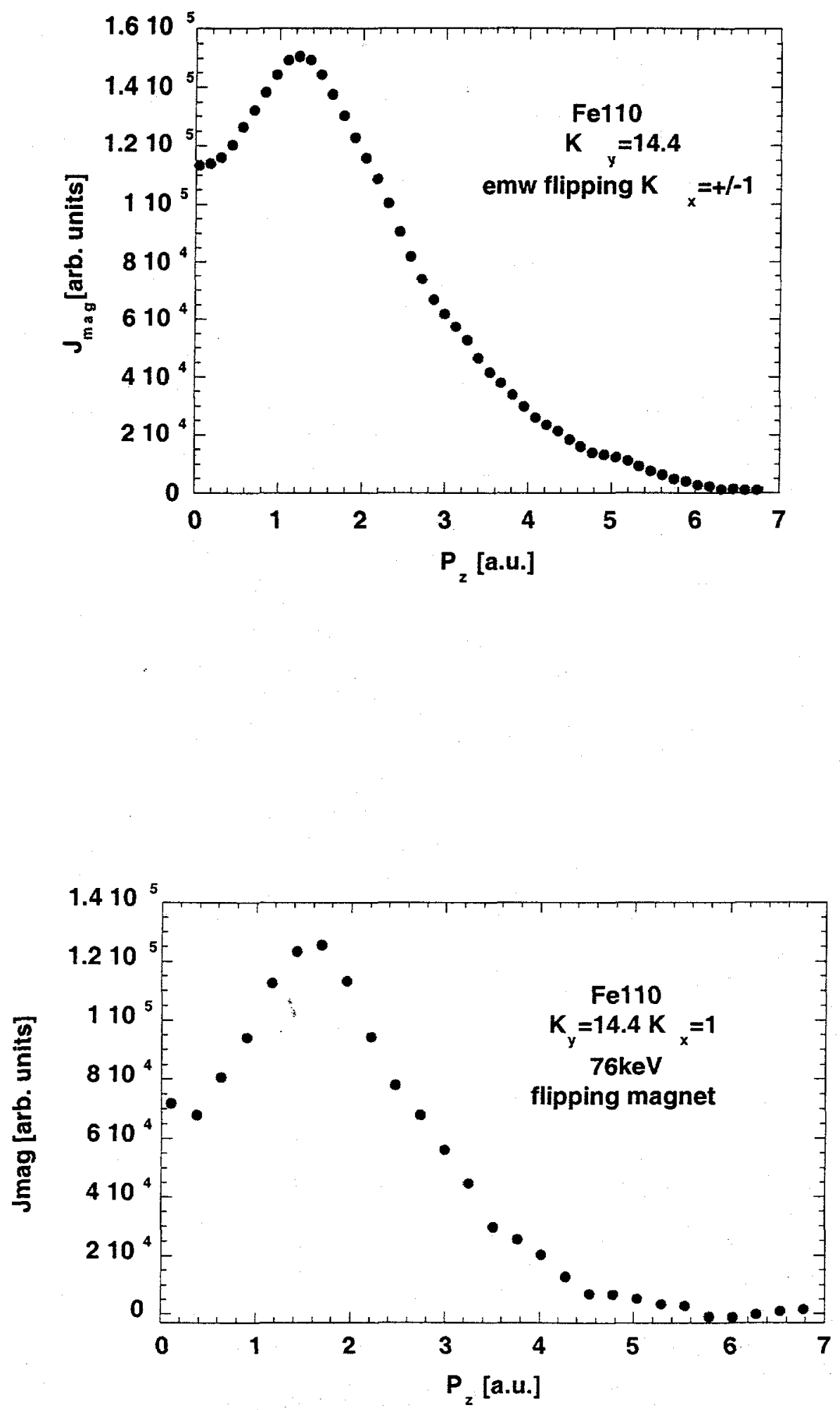


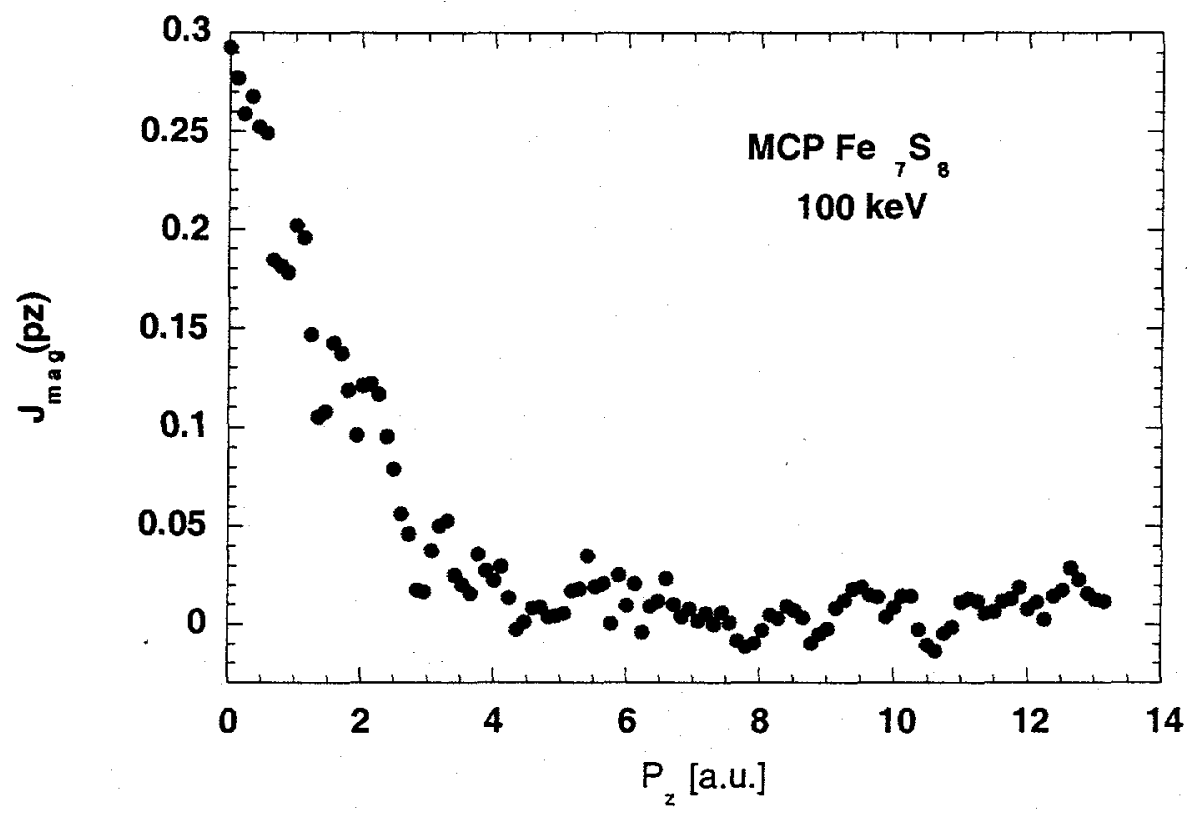

